\title{
44. LEYES DE REFORMA Y DEBATE SOBRE LA REFORMA INSTITUCIONAL EN ITALIA
}

\author{
FULCO LANCHESTER
}

Ordinario Nell'Universitá di Roma "La Sapienza» 


\section{,}




\title{
44. LEYES DE REFORMA Y DEBATE SOBRE LA REFORMA INSTITUCIONAL EN ITALIA
}

POR

\author{
FULCO LANCHESTER
}

Ordinario nell'Universitá di Roma «La Sapienza»

INNOVAZIONI ISTITUZIONALI E SITUAZIONE POLITICA

1. Il ciclo elettorale dei mesi di marzo e di aprile ha confermato la peculiaritá della situazione italiana. Elezioni che dovevano costituire un segnale per la stabilitá o per il cambiamento radicale si sono dimostrate ancora una volta incapaci di fornire un risultato univoco. La loro contradittorietá é evidenziata: dalla incisiva vittoria della "Lega nord" nelle regioni settentrionali e più sviluppate, dove raggiunge percentuali medie superiori al 15\%; dall'indebolimento della Dc, il tradizionale partito pilone, che rimane - nonostante tutto- la prima formazione politica del sistema, distanziando addirittura l'avversario di sempre (il Pds); dal quasi dimezzamento del Pds rispetto al Pci di cui dovrebbe essere l'erede (ma le perdite sono molto inferiori se si conta anche il Partito della rifondazione comunista); dalla fine dell'onda lunga socialista, che tuttavia conserva la sua indispensabilitá come partito cerniera; dalla ulteriore polverizzazione della rappresentanza politica.

In una parola il sistema politico italiano persiste nella sua cronica crisi di transizione delle tradizionali alleanze, mentre il riallineamento del sistema partitico, su cui molti basavano le analisi palingenetiche, risulta non essersi dispiegato in modo sufficiente nè completo. Si può anche sostenere che le più generali tendenze manifestatesi a livello europeo (difficoltà delle sinistre; fine dell'incapsulamento elettorale tipico della contrapposizione tra blocchi) abbiano avuto effetti, ma in modo differenziato e tipicamente condizionato dalla situazione nazionale. 
Simili risultati pongono come primo interrogativo se questa sará veramente la legislatura costituente che molti auspicano o non altro che un periodo di nuova transizione verso nuovi equilibri, nella continuità di una storia nazionale oramai quasi centocinquantenne. In secondo luogo c'è da chiedersi se e quali innovazioni potranno essere introdotte in un panorama politico-istituzionale così complesso, dove alle difficoltà della classe politica corrispondono spinte trasversali che tendono a modificazioni incisive non solo dei meccanismi istituzionali, ma dello stesso regime e della comunità politica.

Qualsiasi prognosi in materia risulta in effetti difficile da formulare, poichè tutti richiedono le innovazioni, ma posizioni di forza e ipotesi istituzionali sono distanti ed insufficienti. La nuova legislatura si apre, infatti, con molte aspirazioni, ma con un deficit di applicabilità delle stesse che non spinge all'ottimismo. Risulta evidente che il mio compito in questa sede non è certo quello di risolvere le questioni poste sul tappeto, quanto quelle di esplicitarle, sulla base di un'analisi che ricostruisca il filo rosso del dibattito in materia di innovazioni istituzionali. Vediamo dunque di esaminare in modo sintetico, ma sistematico i temi impliciti nella questione, ponendo al centro dell'attenzione l'argomento relativo all'adeguamento dell'assetto relativo sia alla forma di governo e al sistema elettorale sia al tipo di Stato attraverso opportune innovazioni.

2. Come è noto il sistema politico-costituzionale italiano funziona in modo anomalo rispetto agli "standards" delle maggiori democrazie occidentali. Si tratta infatti di un ordinamento frammentato politicamente, che contribuisce a generare esecutivi instabili ed inefficienti. Il cattivo funzionamento istituzionale e il permanere di un deficit pubblico di proporzioni non usuali nei paesi occidentali hanno fatto parlare di un ritardo della società politica rispetto allo sviluppo della società civile. Si tratta di una tesi che ha profonde radici e che condanna, con l'assetto istituzionale, anche la classe politica che vi opera. A questa tesi estrema corrisponde l'altra che giustifica la attuale situazione, evidenziando come classe politica ed assetto istituzionale vigenti siano frutto di una società civile frammentata e percorsa da fratture profonde. Secondo questa impostazione, la società civile e la stessa corona ampia della classe dirigente riceverebbe ciò che si merita da istituzioni e soggetti politici fatti a sua immägine é somiglianza.

Una simile polemica riproduce le classiche "querelles" della III Repubblica e del periodo statutario italiano sulla scissione tra paese reale e paese legale e non aggiunge nulla al nostro parco di conoscenże per operare. È forse più pragmatico osservare che se è vero che la dinamica istitu- 
zionale vigente in Italia è frutto delle contraddizioni di una società civile e politica frammentata (che ha acuito con la propria natura bloccata i difetti di sviluppo molto spesso assistenziale e spartitorio), è anche possibile sostenere che le modifiche intervenute nella società civile stessa potrebbero far sopportare alla stessa un corpetto istituzionale dotato de maggiore selettività ed efficienza, sul'esempio di altri ordinamenti europei. Una simile impostazione permette di guardare con maggiore elasticità alle ragioni di lungo e di medio periodo che giustificano la situazione italiana, ma non toglie nulla alle difficoltà di come innovare. I processi di innovazione infrasistemica senza crisi di regime sono infatti lenti e non decisivi, perchè devono fare $\mathrm{i}$ conti con l'esistente e la sua deriva.

Al fine di giustificare simili affermazioni è indispensabile però ricostruire-seppure in modo sintetico-alcuni punti fermi del contesto attuale. In primo luogo deve essere recordato che per il sistema politico-costituzionale italiano costituisce una radicata tradizione la mancanza di istituzioni stabili. Alcuni autori fanno risalire questa situazione addirittura al periodo preunitario (il connubio cavouriano), ma è evidente come le fratture regionali, economiche, religiose e sociali abbiano influenzato la costruzione di un modello di forma di governo parlamentare basata sul trasformismo. (Chiarisco per l'ascoltatore straniero che per trasformismo si intende una pratica della forma di governo parlamentare basata sull'esistenza di maggioranze cangianti e non coese, con lo scivolamento progressivo delle stesse in Parlamento a seconda delle contingenze). Già nell'ultimo ventennio del secolo scorso l'insoddisfazione per il funzionamento trasformistico del sistema politico si traduceva da un lato nella aspirazione di pervenire ad una dinamica di tipo britanico, dall'altro nella costituzione di un vero partito conservatore.

L'estraneità dallo Stato liberale delle componenti cattolica e socialista e la debolezza della borghesia italiana e delle istituzioni liberali nel resistere alla cosiddetta crisi di partecipazione post-bellica si risolsero nel ventennio autoritario, che per alcuni costitui il primo esempio di delega della borghesia italiana nei confronti di una forza politica a lei esterna. II secondo si condensò, dopo il 1947, nella attribuzione alla classe politica cattolica di un mandato oramai più che quarantennale. Nonostante la costituzione del 1948 avesse disegnato una democrazia sociale avanzata con il contributo delle componenti cattoliche, social-comuniste e laiche il sistema istituzionale riprese a funzionare in modo anomalo per la presenza di partiti antisistema nell'ordinamento (parecchi commentatori hanno provveduto a sottolineare sopratutto l'anomalia di un ordinamento caratterizzato dal più forte partito comunista del mondo occidentale). L'assetto istituzionale disegnato dalla Costituente non poteva, dunque, che risentire della paura del tiranno con la scelta di istituzioni deboli, basate su un meccanismo di 
trasformazione dei voti in seggi di tipo speculare (proporzionale) e su una vasta rete di contropoteri (Corte cost., regioni, bicameralismo).

Solo durante il periodo degasperiano, in piena guerra fredda, l'ordinamento è stato, per breve tempo, caratterizzato da un funzionamento stabile della forma di governo parlamentare comparabile a quella britannica. Negli altri periodi è prevalsa la già citata tradizione trasformistica e l'indeterminatezza delle maggioranze, al di là delle formule politiche adottate. D'altro canto gli anni tra il 1948 ed il 1953 non possono essere considerati normali sia per la contrapposizione internazionale ed interna, sia per la tensione istituzionale che comportò la cosidetta legge del premio di maggioranza, primo ed unico tentativo di introdurre un sistema elettorale selettivo all'interno di quest'ordinamento. Quell'ipotesi, funzionale alle esigenze del partito di maggioranza relativa (la Dc), che ricercava la stabilizzazione delle coalizioni attorno a se stessa, costitui un esempio di alternativa istituzionale rispetto all'applicazione della Costituzione del 1948 e all'inserzione progressiva nell'area della maggioranza delle opposizioni.

Dopo il mancato scatto della "legge truffa" l'apertura a sinistra venne preceduta da un periodo di transizione che ipotizzò anche alternative di destra (Tambroni). Gli anni Sesanta si caratterizzarono, invece, per l'alleanza di centro-sinistra con l'ingresso dei socialisti nell'area di governo, ma anche per il fallimento degli scopi riformistici dell'alleanza stessa. Nemmeno gli scopi politici (indebolire il Pci a favore del Psi) vennero raggiunti a alle soglie degli anni Settanta, dopo le elezioni del 1968 che consacrarono la sconfitta del governo Moro, iniziò la grande transizione che avrebbe dovuto portare in un primo tempo all'ingresso del Pci nella maggioranza di governo, superando la conventio ad excludendum, ed in un secondo alla normalizzazione del funzionamento sistemico. Tutti gli anni Settanta si sono giocati attorno al tema dell'affidabilità democratica del Pci, mentre veniva a costruirsi un assetto istituzionale consociativo in cui maggioranza e opposizione convivevano secondo schemi di puro stampo trasformistico.

La crisi economica della seconda metà degli anni Settanta, il terrorismo, l'impasse istituzionale portò a ripensare in modo profondo l'assetto istituzionale. Si comprese alle spalle della tornata elettorale 1976 che il processo di integrazione raggiunto doveva essere accompagnato da una profonda riforma degli stessi assetti istituzionali. In questo dibattito furono particolarmente attive le forze laico-socialiste, che si trovavano allora schiacciate dal bipolarismo Dc-Pci: esse richiedevano l'attivazione di una democrazia dell'alternanza che facesse del sistema italiano qualcosa di più simile alle grandi democrazie europee. Una simile impostazione si concretizzò nel 1979 con la parola d'ordine del leader socialista Craxi della "grande riforma", ma si affievoli -almeno per i socialisti- sia con la pre- 
sidenza dello stesso Craxi, sia con il procedere del dato obiettivo dell'integrazione comunista. Nella IX legislatura molti credettero che il momento dell'innovazione incisiva fosse arrivato con l'istituzione della Commissione per lo studio delle riforme istituzionali presieduta dall'on. Bozzi ed alcuni ritennero addirittura, di potere operare un parallello tra la stessa e la Commissione del 75 , che operò durante il periodo costituente. Un simile sbaglio venne subito smentito sia dai Comitati limitati della Bozzi sia dalla persistenza dei voti contrapposti tra i partiti. In quel periodo, mentre il Psi risultò praticamente assente dal dibattito perchè attratto dell'effetto Presidenza del Consiglio, la Dc continuava a prospettare riforme stabilizzatrici delle alleanze per mezzo di premi alle coalizioni ed il Pci risultava sostancialmente sospettoso nei confronti di qualsiasi innovazione che avesse preceduto il problema della caduta della sua esclusione dall'area di Governo.

Pochi anni dopo sia Dc che Pci evidenziarono progetti sempre più incisivi di trasformazione istituzionale, mentre il Psi non poteva che assumere una posizione difensiva a causa delle sue dimensioni che lo avrebbero sfavorito comunque. Da un simile panorama è venuta fuori la classica situazione di blocco dell'innovazione in cui tutti i soggetti impediscono agli altri il movimento. La battaglia istituzionale e la strategia alternativistica del Pci (ora Pds) si è quindi sposata con la tendenza alla costituzione di un partito trasversale per le riforme incisive sopratutto di tipo elettorale. II movimento referendario di Mariotto Segni ha intrapreso in questi anni con uno spettro molto ampio una battaglia per l'introduzione di meccanismi elettorali selettivi che ha ereditato dal partito radicale l'utilizzazione eversiva per il rinnovamento del referendum abrogativo. La sent. n. 47/1991 che ha dichiarato ammissibile solo il referendum sulla preferenza unica e il successo della proposta abrogazionista nella votazione del 9/10 giugno dell'anno scorso sono stati solo i primi episodi di una battaglia che ha visto la ripresentazione dei quesiti, la riproposizione della questione per l'anno prossimo e la costituzione di un "patto" referendario tra i candidati alle ultime elezioni politiche. I circa centocinquanta deputati (su novecentoquarantacinque) legati al patto Segni potranno giocare un ruolo non indifferente nella prossima legislatura, che non nasce sotto il segno della chiarezza.

3. In effetti gli ultimi due anni di storia italiana sono stati anche caratterizzati da una crisi istituzionale intensa derivante dalla perdita di valore strategico delle tradizionali alleanze e dalla tendenza alla ristrutturazione dello stesso sistema partitico. Tutti gli istituti costituzionali sono stati messi sotto tensione ed alla prova. In particolare, mentre nel corso della legislatura sono stati operati gli accostamenti istituzionali massimi compatibili con la natura coalizionale ed instabile del sistema, si è venuta producendo 
un'attività di stimolo non convenzionale da parte del Capo dello Stato. Francesco Cossiga sulla base di una complessa serie di motivazioni personali e politiche, ha operato come maieuta per il mutamento. Egli ha dichiarato più volte (ricordo a questo proposito il messagio al parlamento del giugno dell'anno scorso) che il mutamento è necessario non solo a causa delle profonde trasformazioni avvenute nel contesto interno ed internazionale, ma anche si è posto il compito di operare in prima persona per la transizione verso il nuovo. II continuo riferimento all'esperienza della fine della IV Repubblica individua una proposta strategica in cui Cossiga dovrebbe sicuramente ricoprire il ruolo di Coty. D'accordo con una simile posizione è anche l'ideologo della Lega Gianfranco Miglio, politologo e già preside della Facoltà di Scienze politiche dell'Università Cattolica di Milano, il quale ha prefigurato il mutamento per spallate successive. La Lega nord di Umberto Bossi, che nelle regioni settentrionali del paese ha ottenuto un successo imponente, divenendo in molte località il primo partito ed indebolendo fortemente le formazioni tradizionali (ed in modo particolare la Democrazia cristiana), pensa di incrementare la propria forza con le elezioni amministrative del 1995, dando vita alla Italia federale.

II sistema partitico che per quarantacinque anni ha caratterizzato il regime politico si trova oggi in affanno per un assalto esterno che indebolisce oltremodo l'arco costituzionale. Se è vero infatti che l'ordinamento italiano dal 1945 ad oggi è stato caratterizzato dalla scelta in media di un terzo dell'elettorato per formazioni antisistema, è anche vero che è la prima volta che più di un quarto dello stesso non si riconosce più nei partiti che furono alla base del patto costituzionale. È questo un segno indubbio della pericolosità della situazione attuale e della necessità di percorrere nuove strade rispetto a quelle tradizionali.

In una simile situazione il Capo dello Stato ha utilizzato a tutto campo i propri poteri di stimolo e di esternazione, fino ad incappare nelle critiche più feroci e nello stesso procedimento di «impeachment". In proposito direi che l'attività di Cossiga non può essere tanto criticata per le sue singole estrinsecazioni, quanto per il complesso della stessa. Essa ha sicuramente messo in difficoltà sia la strategia socialista volta all'introduzione dell'elezione diretta del Capo dello Stato sia la linea continuista della Dc, ma sopratutto ha dimostrato come nello stesso Cossiga viva una interpretazione del suo ruolo di tipo costituzionale puro difficilmente inseribile all'interno del la forma di governo vigente.

Dopo le lezioni di aprile le polemiche sul ruolo del Presidente della Repubblica e sulle procedure di messa in stato d'accusa dello stesso, sull'immobilismo sostanziale di una maggioranza raggelata dalla paura dell'ascesa leghista e sul ruolo del Capo dello Stato sono oramai molto 
meno attuali in un momento in cui si discute, in una situazione estremamente fluida:
a) sui percorsi per arrivare alla innovazione istituzionale;
b) sui contenuti e sui limiti delle innovazioni possibili;
c) sulla praticabilità e capacità riformistiche delle innovazioni pro- gettate.

4. A questo punto è necessario lasciare la cornice politica per chiarire alcuni temini più strettamente istituzionali della questione.

In primo luogo quello relativo ai percorsi per arrivare alla innovazione delle istituzioni ed in particolare del funzionamento della forma di governo.

$\grave{E}$ una oramai radicata tradizione del dibattito in materia di istituzioni differenziare tra innovazioni che esigono la revisione costituzionale e innovazioni che invece possono essere assunte per legge ordinaria o addirittura per convenzione (ossia accordo tacito tra i soggetti politicamente rilevanti). Innovazioni relative alla forma di governo o al tipo di Stato di solito non possono prescindere della revisione della Costituzione di cui all'art. 138 Cost. e quindi ricadono in un procedimento aggravato e faticoso.

L'art. 138 della Cost. prevede, infatti, due approvazioni differenziate in entrambe le Assemblee, con un intervallo non minore di tre mesi e con la maggioranza assoluta dei componenti nella seconda votazione (se nel corso di questa deliberazione non è stata raggiunta la maggioranza dei due terzi, si può dare luogo ad un referendum popolare).

Come è intuibile una simile procedura non può che rendere difficile se non impossibile la decisione in un ordinamento frammentato come quello italiano. Ed è per questo che suscitò da un lato meraviglia e dall'altro preoccupazione la discussione avvenuta sopratutto durante l'ultima crisi di governo sulla sospensione temporanea della validità dell'art. 138 o sulla sua modificazione. Presidente della Repubblica e Presidente del Consiglio si dissero favorevoli assieme ad altri esponenti del mondo politico ad una simile soluzione, mentre altri lanciarono grida d'allarme. Ciò fece rinascere il dibattito sul rapporto tra Costituzione e leggi costituzionali e sui conseguenti limiti impliciti alla revisione e sul ruolo della Corte costituzionale nel processo di revisione stesso. Ad alcuni tutto questo attivismo ri- 
formatore dette l'impressione che si trattasse di un givoco delle parti, poichè risultava intuitivo che nessuno avrebbe rinunciato al proprio potere di interdizione e che il problema veniva posto in maniera provocatoria al fine di non cambiare nulla.

Nel dibattito politico-pragmatico sono state proposte anche innovazioni di tipo "convenzionale", basate sull'accordo dei soggetti politicamente rilevanti: di qui, ad esemplo, l'idea dei patti di legislatura informali o quella di governi di tecnici e di transizione condotti da Presidenti del Consiglio che escludessero ogni influenza partitica appoggiandosi sulla lettera dell'art. 92 Cost. Tutte idee inapplicabili, se si pensa che la natura intrinseca delle convenzioni costituzionali è quella di comportamenti ripetuti in situazioni stabilizzate da parte dei soggetti interessati, sottoposti a sanzioni rilevanti di tipo relazionale. Ciò spiega come le proposte in materia (da quelle meno recenti di Galeotti a quelle sul diritto costituzionale provvisorio di Manzella) siano state lasciate cadere in favore di più stabili innovazioni legislative.

Le innovazioni legislative più richieste, applicabili senza revisione costituzionale e per legge ordinaria, sono - invece- principalmente quelle elettorali. Come è noto in Italia la normativa relativa al sistema elettorale in senso stretto non è stata costituzionalizzata: purtuttavia essa si connette in modo inscindibile al regime politico e diviene comprensibile come essa abbia assunto una caratteristica di immodificabilità sostanzialmente costituzionale. Dopo l'episodio della legge "truffa" qualsiasi discussione sul sistema elettorale in senso stretto è stata considerata impossibile ed, anzi, la normativa in questione è venuta a schiacciarsi sulla convenzione proporzionalistica. Risulta quindi palmare la difficoltà di percorrere una simile strada, anche se i progetti in materia si sono affollati in questi ultimi anni in stretta correlazione con il mutamento della politica istituzionale comunista (oggi pidiessina) e con l'espandersi del movimento referendario di cui parlerò subito di seguito.

Per rompere la logica bloccata dei veti partigiani si è formata in Italia una coalizione trasversale per l'innovazione elettorale (ma quale?) che utilizza l'arma del referendum abrogativo. La prima fase della battaglia si è svolta l'anno scorso sia davanti alla corte costituzionale che ha dichiarato ammissibili ai sensi dell'art. 75 cost. solo uno dei tre quesiti referendari proposti, sia davanti al Corpo elettorale che investito della questione nel giugno 1991 ha abrogato la normativa sulla preferenza unica. II fronte referendario, che raccoglie - tra gli altri- membri della Dc, del Pds, delle organizzazioni cattoliche, della Cofindustria e della società civile, non soltanto ha ripresentato i quesiti dichiarati innammisibili l'anno scorso, ma ha provveduto - prima delle elezioni politiche - alla formulazione di un "patton tra i candidati alle elezioni politiche. In Parlamento sono quindi stati 
eletti circa centocinquanta rappresentanti "pattisti», che si sono impegnati a perseguire - al di là di ogni disciplina di partito- l'obiettivo della riforma elettorale. II sistema politico si trova d'altro canto difronte alla minaccia di un eventuale referendum sul sistema elettorale che trasformerebbe la legge del senato in sostanzialmente maggioritaria e che farebbe divenire totalmente maggioritaria quella per le amministrazioni comunali. Lo scopo dei pattisti non è sicuramente quello di mantenere una simile normativa, ma di convincere la classe politica ad innovare.

In questa prospettiva è possibile che il Parlamento pur di non dover rischiare approvi una nuova normativa elettorale che aumenti la selettività del sistema, ma è anche pensabile che -impossibilitato a innovare- si affidi o al giudizio di inammissibilità della Corte costituzionale o a una improbabile ripetizione della farsa dell'anno scorso con l'invito di andare al mare (si badi bene che è legittimo astenersi, ma è miope invitare su un soggetto così controverso l'elettorato a farlo).

Mentre i progetti in materia elettorale si moltiplicano e si pensa anche riformare incisivamente l'articolazione regionalistica dell'ordinamento (accogliendo in parte le richieste della Lega), le prospettive reali dell'innovazione non sono assolutamente migliorate a circa un mese dalla consultazione d'aprile. L'impasse sembra, dunque, persistere: le scelte sono ancora nebulose e si situano faticosamente, ma senza vera decisione, tra la continuità con il passato e la transizione verso nuovi equilibri. 


\section{NOTA BIBLIOGRAFICA}

A fini di mero orientamento a senza alcuna pretesa di completezza, qui di seguito vengono indicate alcune opere pubblicate in Italia in materia di innovazione istituzionale.

1) Sulle tendenze di fondo della forma di governo in Italia, v.: E. CHELI, Costituzione e sviluppo delle istituzioni in Italia. Bologna, II Mulino, 1978; C. CHIMENTI, Introduzione alla forma di governo italiana, Rimini, Maggioli, 1985; i saggi di OdINA, Amato-Bruno, Rotell puglicati sui numeri 1 e 2 di Quaderni costituzionali, 1981 e la "Tavola rotonda" su "La forma di governo nell'Italia odierna" (contributi di Amato, Barbera, BalBoni, Calandra, Cheli, Elia, Grisolia, lanchester, Long, Manzella, Onida, Paladin, Pegoraro, Tosı), pubblicata sul n. 2/1991 di questa stessa rivista.

2) Sul dibattito sulle riforme istituzionali, $v$.: G. Amato, Una repubblica da riformare, /l dibattito sulle istituzioni in Italia dal 1975 ad oggi, Bologna, II Mulino, 1980; G. PAsouIno, Degenerazioni e riforme dei partiti, Bari, Laterza, 1982; Grupo dI MILANo, Verso una nuova costituzione, Milano, Giuffré, 1983, 2 tomi. AA.Vv., / Parlamento tra crisi e riforma, Milano, Franco Angeli, 1985; P. ArMAROLl, L'introvabile governabilità: le strategie istituzionali dei partiti dalla costituente alla Commissione Bozzi, Padova, Cedam, 1986; G. Pasouino, Restituire lo scettro al principe. Proposte di riforma istituzionale, Bari, Laterza, 1986; V. AtRIPALDI-F. FicheRA, Dalla grande riforma alla politica delle istituzioni, Padova, Cedam, 1986; F. LANCHESTER, Votazioni, sistema politico e riforme istituzionali, Roma, Bulzoni, 1987; AA.W., Altra repubblica? Perchè come quando, a cura di J. Jacobelli, Bari, Laterza, 1988; II cittadino come arbitro, a cura di R. Ruffilli-P. A. Capotosti, Bologna, II Mulino, 1988; La lenta marcia nelle istituzioni: i passi del Pci, a cura di G. Pasquino, Bologna, II Mulino, 1988; F. TERESI, Le riforme istituzionali tra governabilità, partecipazione e trasparenza. Materiali di studio, Torino, Giappichelli, 1989; F. LANCHESTER, Rappresentanza, responsabilità e tecniche di espressione del suffragio, Roma, Bulzoni, 1990; G. MıGLı, Una Costituzione per i prossimi trent'anni. Intervista sulla terza Repubblica, a cura di M. Staglieno, Bari, Laterza, 1990; G. PAsouino, La repubblica dei cittadini ombra, Milano, FeltrineIli, 1991; C. Fusaro, Guida alle riforme istituzionali, Soveria Mannelli, Rubet- 
tino, 1991; AA.VV., "Le "forme della democrazia"", in Democrazia e diritto, 1991, n. 4; A. BARBERA, Una riforma per la Repubblica, Roma, Editori Riuniti, 1991; G. SARToRi, Seconda Repubblica? Si, ma bene, Milano, Rizzoli, 1992; S. Messina, La grande riforma. Uomini e progetti per una nuova repubblica, Bari, Laterza, 1992; G. MıgLIo, Come cambiare. Le mie riforme, Milano, Mondadori, 1992.

3) Per le discussioni istituzionali v. Commissione parlamentare per le riforme istituzionali-IX legislatura, Atti, 3 voll., pubblicato dalla Camera dei deputati e dal Senato della Repubblica; SenatTo deLLA RePubblica, La riforma del bicameralismo in Senato, intr. di G. Spadolini, Roma, 1990; $\mathrm{C}_{\mathrm{A}}$ MERA DEI DePUTATI, La Costituzione e le riforme istituzionali. II dibattito alle Camere sul messagio del Presidente della Repubblica (23-25 luglio 1991), Roma, 1991.

4) Sul rapporto maggioranza e opposizione in Parlamento e la modifica incrementale dei regolamenti del 1971 durante gli anni Ottanta $v$. A. Manzella, I/ Parlamento, Bologna, II Mulino, 1991 (nuova ed. rispetto a quella del 1977 con cui necessario confrontarla) e il n.2/1991 di Quaderni costituzionali su /l Parlamento a vent'anni dai regolamenti del 1991 (con contributi, tra gli altri, di CotTA, FloRIDIA, SiCARDI, DE CARO, LIPPOLIS, BarRetTon Arleri, Meschino, Manetti, Costanzo). Sulla legge di riforma della Presidenza del Consiglio dei Ministri v. A. MANZELLA, "Osservazioni sulla legge $n$. 400/1988 sulla presidenza del Consiglio dei Ministri", in Quaderno, n.1 dell'Associazione per gli studi e le ricerche parlamentari, Milano, Giuffrè, 1991, p. 95 ss.

5) Per i problemi relativi al sistema elettorale v.: F. LANCHESTER, Sistemi elettorali e forma di governo, Bologna, II Mulino, 1981; D. FISICHELLA, Elezioni e democrazia, Bologna, II Mulino, 1982; Materiali per la riforma elettorale, a cura di R. Ruffilli, Bologna, II Mulino, 1987; L. TENTONI, Gli strumenti per cambiare. Viaggio nei sistemi elettorali, Roma, Acropoli, 1991; M. Luciani, II voto e la democrazia. La questione delle riforme elettorali in Italia, Roma, Editori Riuniti, 1991.

6) Per la vicenda del referendum abrogativo ed il suo uso nel cambiamento istituzionale v.: CAMERA DEI DEPUTATI, // referendum abrogativo in Italia. Le norme, le sentenze, le proposte di modifica, Roma, 1981; i saggi pubblicati sul n. 2/1985 di Quaderni costituzionali; il volume su I referendum elettorali. Seminario di studio e documentazione, a cura di F. LANCHESTER, Roma, Bulzoni, 1992; M. LUCIANI, "ll referendum impossibile", in Quaderni costituzionali, 1991, n. 3, pp. 509 ss. 